\title{
What Do Young Brown Dwarfs Tell Us About Exoplanets?
}

\author{
Katelyn N. Allers ${ }^{1}$, Michael C. Liu ${ }^{2}$ and Trent J. Dupuy ${ }^{3}$ \\ ${ }^{1}$ Department of Physics and Astronomy, Bucknell University, Lewisburg, PA 17837, USA \\ email: k.allers@bucknell.edu \\ ${ }^{2}$ Institute for Astronomy, University of Hawaii, 2680 Woodlawn Drive, Honolulu, HI 96822, \\ USA \\ ${ }^{3}$ Department of Astronomy, The University of Texas at Austin, 2515 Speedway C1400, Austin, \\ TX 78712 , USA
}

\begin{abstract}
In recent years, all-sky surveys have uncovered a new and interesting population of young $(\approx 10-200 \mathrm{Myr})$, nearby substellar objects. Many of these objects have inferred masses and temperatures that overlap those of directly imaged exoplanets. These young brown dwarfs provide valuable analogs to young, dusty exoplanets in a context where detailed spectroscopic observations across a broad range of wavelengths and at high $\mathrm{S} / \mathrm{N}$ are possible. How do the temperatures inferred by atmospheric models and evolutionary models compare? Can we determine the formation mechanism of a young planetary-mass object? How well do we understand the role that disequilibrium chemistry and dust clouds play in the atmospheres of these objects? We review the successes and challenges in determining the fundamental properties (mass, $\log (g)$, effective temperature) of young substellar objects, both brown dwarfs and gas-giant exoplanets.
\end{abstract}

Keywords. (stars:) brown dwarfs, infrared:stars, infrared:planetary systems

\section{Introduction}

Studies of directly-imaged exoplanets (e.g., Marois et al. 2008; Lagrange et al. 2009), planetary-mass companions (e.g., Gauza et al. 2015; Artigau et al. 2015) and free-floating planetary-mass objects (e.g.; Liu et al. 2013b; Gizis et al. 2012) share a common goal: determination of fundamental properties (mass, $\log (\mathrm{g})$, effective temperature).

The spectra of young, directly-imaged exoplanets are well matched by young field brown dwarfs (e.g., Chilcote et al. 2015). Recently, young brown dwarfs with similar colors and magnitudes as directly-imaged exoplanets have been discovered (Liu et al. 2013b, Gauza et al. 2015). Given that detailed followup spectroscopy is possible for a large population of young field brown dwarfs, to what extent can these objects be used as analogs to extrasolar planets? Can we develop a unified picture of young, planetarymass objects that includes both free-floating objects, wide companions, and exoplanets?

\section{Near-IR Spectroscopic Age Determinations}

Given the mass-luminosity-age degeneracy for substellar objects (brown dwarfs and planetary-mass objects), determination of age is critical for estimating their fundamental properties. In various presentations at this symposium, methods for determining the ages of young stars have been discussed. For low-mass stars, the detection of lithium and/or the position of an object above the zero age main sequence on an H-R diagram are the standard methods for determining age. Neither of these methods, however, can determine the age of brown dwarfs or directly-imaged exoplanets. First, most brown dwarfs $\left(<65 M_{\mathrm{Jup}}\right)$ and all planetary-mass objects do not achieve high enough core 


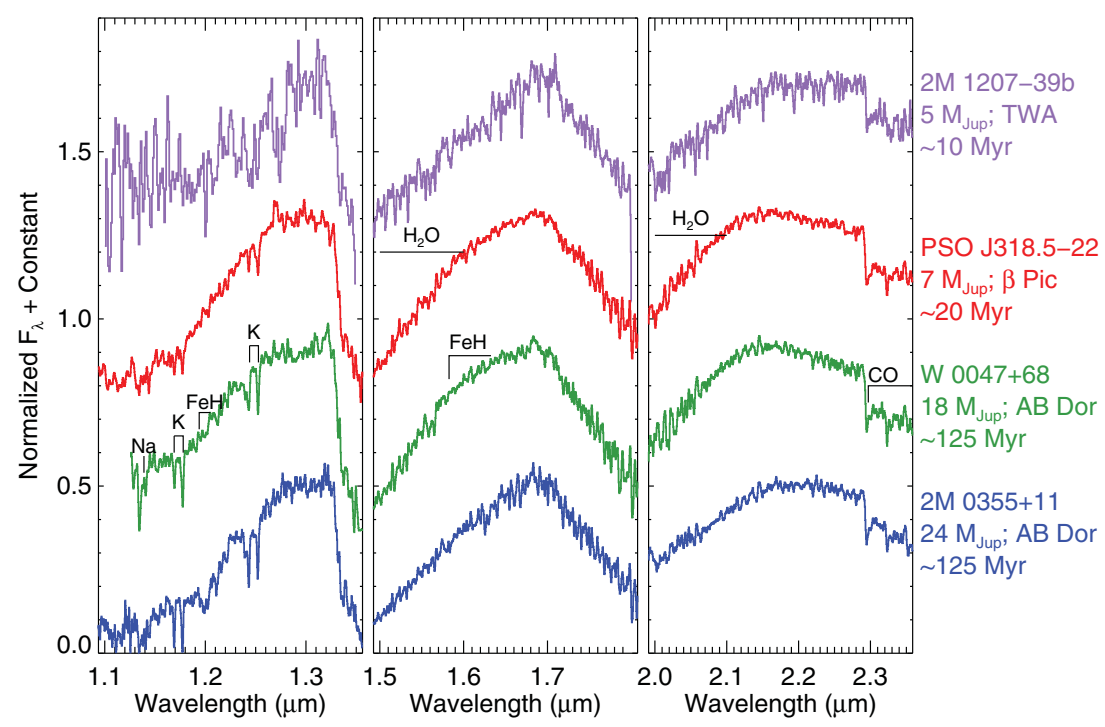

Figure 1. Comparison of the spectra of young brown dwarfs and planetary-mass objects that are kinematically linked to known young moving groups. Despite having similar masses and ages, the spectra of PSO J318.5-22 (Liu et al. 2013b) and 2M 1207-39b (Patience et al. 2010) are not the most similar. The free-floating brown dwarfs, $2 \mathrm{M} 0355+11$ and $\mathrm{W} 0047+68$ are both members of the $\sim 125 \mathrm{Myr}$ old AB Dor moving group (Liu et al. 2013a, Gizis et al. 2015), yet have quite disparate spectra (Allers \& Liu 2013), despite their similar masses.

temperatures to destroy lithium. Second, recent studies indicate that young, ultracool brown dwarfs are fainter than expected, having similar luminosities as their older field dwarf counterparts of the same spectral type (e.g. Liu et al. 2013a).

In contrast to most directly imaged planets, young brown dwarfs are accessible to detailed spectroscopic observations. Cruz et al. (2009) developed a classification scheme for the optical spectra of young brown dwarfs. Allers \& Liu (2013) presents a corresponding classification system using near-IR spectra. Both of these studies classify the gravitysensitive spectral features into rough age bins of $\lesssim 30 \mathrm{Myr}$ (labeled as $\gamma$ in the optical and VL-G in the near-IR) and 30-200 Myr ( $\beta$ in the optical and INT-G in the near-IR). Both studies emphasize that objects of the same spectral classification can have varying $J-K$ colors. Allers \& Liu (2013) also note that objects of the same spectral type that are tied to same YMG can display significant differences in their gravity-sensitive (youth) spectral features in the near-IR. An illustration of this is shown in Figure 1. In terms of mass and age, 2M 1207-39b and PSO J318.5-22† are remarkably similar. When looking at the spectral shapes and gravity sensitive features, PSO J318.5-22 is much more similar to W $0047+68$, a markedly older object. Likewise, the spectrum $2 \mathrm{M} 1207-39 \mathrm{~b}$ most closely resembles $2 \mathrm{M} 0355+11$ (Faherty et al. 2013), an older, more massive object, which is over 2 magnitudes brighter than $2 \mathrm{M} 1207-39 \mathrm{~b}$ in $J$ band.

Even objects that lie at the same position on a CMD can display disparate spectra. The recently discovered planetary-mass object VHS 1256-1257B (Gauza et al. 2015) has colors and magnitudes that agree (to within the uncertainties) with the directly-imaged exoplanet, HR 8799b. Figure 2 shows the spectra of these two objects. Despite having incredible similarity in color and magnitude, these two objects have quite different spectral shapes. Overall, observations of young brown dwarfs and directly-imaged exoplanets

$\dagger$ The recently-measured radial velocity of PSO J318.5-22 confirms that it is a member of the $\sim 20$ Myr-old $\beta$ Pictoris moving group (Allers et al., in prep). 


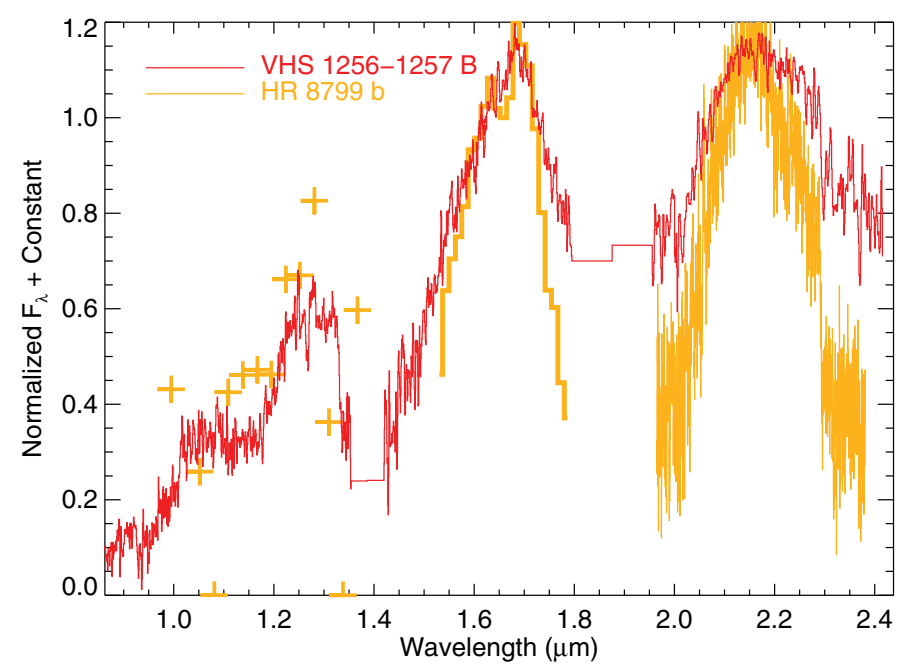

Figure 2. Comparison of the spectrum of VHS 1256-1257B (Gauza et al. 2015) and HR 8799b (Barman et al. 2011, 2015; Oppenheimer et al. 2013). The $J-H$ color and absolute $J$-band magnitude of VHS 1256-1257B agree (to within the uncertainties) with photometry of the exoplanet, HR 8799b. The spectra, however, have very different spectral shapes. In particular, the $K$-band spectrum of $\mathrm{HR} 8799 \mathrm{~b}$ shows deeper $\mathrm{H}_{2} \mathrm{O}$ and $\mathrm{CO}$ absorption than seen in the spectrum of VHS 1256-1257B. In addition, HR 8799b has (weak) methane absorption present in its $K$-band spectrum, whereas VHS 1256-1257B does not.

point to diversity in near-IR spectral morphologies, even among objects of the same color, luminosity, and/or age.

\section{Atmospheric and Evolutionary Models}

Based on the spectral diversity seen for objects of similar masses and ages, it is clear that one cannot determine accurate ages and masses for planetary-mass objects from spectra alone. For the handful of planetary-mass objects that are members of young moving groups, one can estimate their masses using the model isochrone for the age of the group. One caution is that evolutionary models have not been tested at such young ages by observations. Indeed, the few available tests at older ages ( $\approx 0.5-1.0$ Gyr) find systematic errors in the model-predicted luminosities and masses at the level of a factor of $\approx 2$ and $\approx 25 \%$, respectively, likely due to the effect of cloud evolution (Dupuy et al. 2009, 2014).

Effective temperatures $\left(\mathrm{T}_{\text {eff }}\right)$ for ultracool dwarfs can be computed using evolutionary models, given the objects' measured bolometric luminosities and estimated ages (e.g., Golimowski et al. 2004). Early discoveries of ultracool companions to young stars suggested a systematic difference between the temperatures of young objects compared to old objects of the same spectral type (e.g., Metchev \& Hillenbrand 2006; Luhman et al. 2007; Dupuy et al. 2009). The HR 8799 planets have brought this effect to the forefront, given the sharp discrepancy between their cool temperatures $(\approx 1000 \mathrm{~K})$ inferred from evolutionary models and their very red, methane-poor spectral energy distributions characteristic of hotter field objects (e.g., Marois et al. 2008; Bowler et al. 2010; Barman et al. 2011; Marley et al. 2012). This discrepancy is now seen in free-floating objects in the field as well, as shown by the young ( $\approx 10-20$ Myr) late-L dwarf PSO J318-22 (Liu et al. 2013). Figure 3 compiles all the temperature results to date derived from evolutionary models, including a greatly expanded sample of temperatures from the Hawaii 


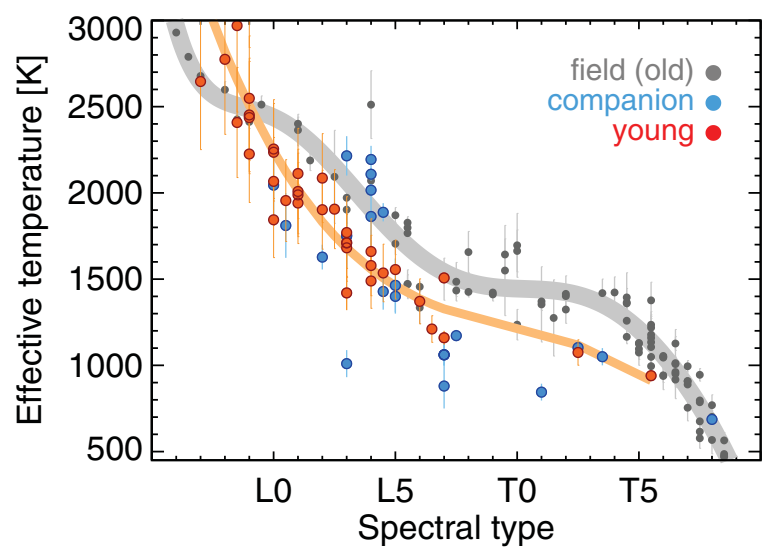

Figure 3. Summary of $\mathrm{T}_{\mathrm{eff}}$ as a function of spectral type for ultracool dwarfs. Field (old) objects (grey) and young companions (blue) are from the calculations and compilation by Bowler et al.(2013), with additions from Naud et al.(2014) and Gauza et al.(2015). Young field objects (orange) are primarily from Liu et al.(in prep), with additional objects from Liu et al.(2013), Gizis et al.(2015), and Gagne et al.(2015). Polynomial fits to the old and young samples are shown as thick colored lines. The young sequence for $\mathrm{L}$ and $\mathrm{T}$ dwarfs runs $\approx 200-300 \mathrm{~K}$ cooler than the old sequence.

Infrared Parallax Program (Dupuy \& Liu 2012) for young objects (Liu et al., in prep). We see now the young field $\mathrm{L}$ and $\mathrm{T}$ dwarfs have a systematic offset relative to the field objects of $\approx 200-300 \mathrm{~K}$ cooler, while the young late-M dwarfs tend to run hotter than the field objects. A similar offset appears to be present between young companions and old field objects though less distinct (see also Bowler et al. 2013), perhaps reflecting the more heterogeneous spectral typing of companion discoveries or (more speculatively) the possibility that young companion and young field objects behave differently.

How do the $\mathrm{T}_{\text {eff }}$ 's determined by fitting model atmospheres compare to the $\mathrm{T}_{\text {eff }}$ 's determined from evolutionary models? Figure 4 shows the best fit atmospheric models to the near-IR spectrum of PSO J318.5-22. As discussed in Liu et al. (2013b) and Gizis et al. (2015), current atmospheric models for young brown dwarfs and planetary-mass objects with L-type spectra overpredict their $\mathrm{T}_{\text {eff }}$ 's . Indeed, if young, planetary-mass objects had the $\mathrm{T}_{\text {eff }}$ 's inferred by atmospheric models, their radii would necessarily be implausibly small $\left(<1 \mathrm{R}_{\text {Jup }}\right.$ ) to match their observed luminosities. The $\mathrm{T}_{\text {eff }}$ 's derived from evolutionary and atmospheric models become more divergent for younger and lowermass objects (Gizis et al. 2015). Recent advances in atmospheric models, including treatment of clouds and disequilibrium chemistry are likely to provide better fits (see contribution by M. Marley). The combination of issues associated with current model atmospheres as well as the diversity seen in the observed spectra of young planetary-mass objects suggests that $\mathrm{T}_{\text {eff }}$ 's determined from fitting model atmospheres to near-IR spectra are unreliable and corresponding surface gravity determinations even moreso.

\section{Conclusions}

- Spectral diversity is the norm in the young substellar regime, even for objects of similar, mass, age, and/or position on a color-magnitude diagram.

- Young substellar objects (brown dwarfs and exoplanets) have cooler effective temperatures than field brown dwarfs of the same spectral type, both for low-gravity objects in the field and those found as companions to young stars. 


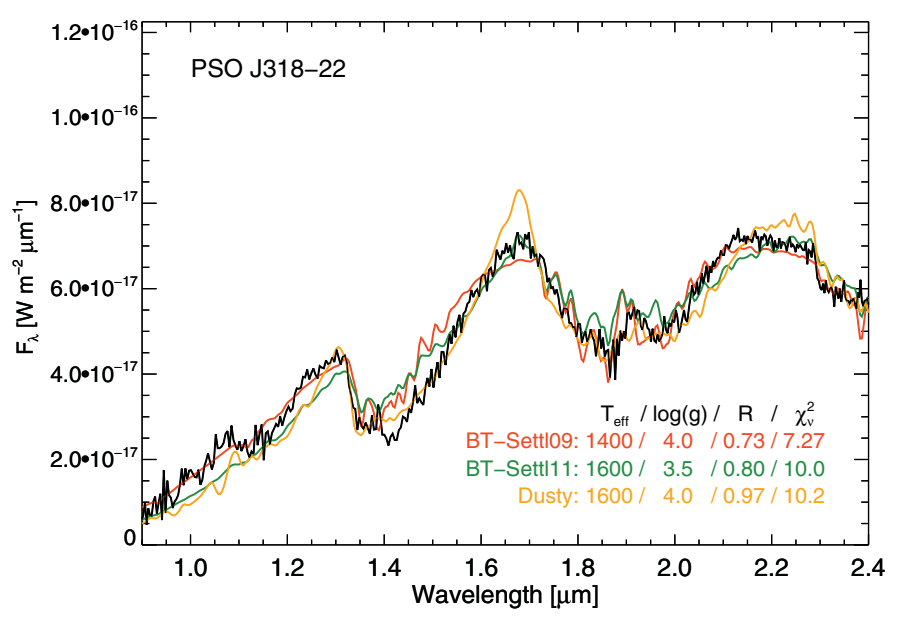

Figure 4. Comparison of the spectrum of PSO J318.5-22 (black; Liu et al. 2013b) to best fit model atmospheres (Allard et al. 2001, 2012), as determined from $\chi^{2}$ minimization. Since PSO J318.5-22 has a known distance, the scaling factor needed to match the flux level of the model to the observed spectrum $\left(=R^{2} / d^{2}\right.$ where $R$ is the radius and $d$ is the distance) provides an estimate of PSO J318.5-22's radius. The best-fit models yield implausibly small radii. As a member of the $\approx 10-20$ Myr old $\beta$ Pictoris moving group, evolutionary models predict a radius of $1.56 R_{\mathrm{Jup}}$ and effective temperature of $1190 \mathrm{~K}$ for PSO J318.5-22, significantly cooler than the temperatures of the best-fit atmospheric models.

- The effective temperatures of young brown dwarfs and directly-imaged exoplanets are overpredicted by atmospheric models for L-type objects.

\section{References}

Allard, F., Hauschildt, P. H., Alexander, D. R., Tamanai, A., \& Schweitzer, A. 2001, ApJ, 556, 357

Allard, F., Homeier, D., Freytag, B., \& Sharp, C. M. 2012. Atmospheres From Very Low-Mass Stars to Extrasolar Planets. EAS Publications Series 57, 3-43

Allers, K. N. \& Liu, M. C. 2013, ApJ, 772, 79

Artigau, É., Gagné, J., Faherty, J., et al. 2015, ApJ, 806, 254

Barman, T. S., Konopacky, Q. M., Macintosh, B., \& Marois, C. 2015, ApJ, 804, 61

Barman, T. S., Macintosh, B., Konopacky, Q. M., \& Marois, C. 2011, ApJ, 733, 65

Bowler, B. P., Liu, M. C., Dupuy, T. J., \& Cushing, M. C. 2010, ApJ, 723, 850

Bowler, B. P., Liu, M. C., Shkolnik, E. L., \& Dupuy, T. J. 2013, ApJ, 774, 55

Chilcote, J., Barman, T., Fitzgerald, M. P., et al. 2015, ApJL, 798, L3

Cruz, K. L., Kirkpatrick, J. D., \& Burgasser, A. J. 2009, AJ, 137, 3345

Dupuy, T. J., Liu, M. C., \& Ireland, M. J. 2009, ApJ, 699, 168

Dupuy, T. J., Liu, M. C., \& Ireland, M. J. 2014, ApJ, 790, 133

Dupuy, T. J. \& Liu, M. C. 2012, ApJS, 201, 19

Faherty, J. K., Rice, E. L., Cruz, K. L., Mamajek, E. E., \& Núñez, A. 2013, AJ, 145, 2

Gagné, J., Burgasser, A. J., Faherty, J. K., et al. 2015, arXiv:1506.04195

Gauza, B., Béjar, V. J. S., Pérez-Garrido, A., et al. 2015, ApJ, 804, 96

Gizis, J. E., Allers, K. N., Liu, M. C., et al. 2015, ApJ, 799, 203

Gizis, J. E., Faherty, J. K., Liu, M. C., et al. 2012, AJ, 144, 94

Golimowski, D. A., Leggett, S. K., Marley, M. S., et al. 2004, AJ, 127, 3516

Lagrange, A.-M., Gratadour, D., Chauvin, G., et al. 2009, A\&SA, 493, L21

Liu, M. C., Dupuy, T. J., \& Allers, K. N. 2013a, Astronomische Nachrichten, 334, 85

Liu, M. C., Magnier, E. A., Deacon, N. R., et al. 2013b, ApJL, 777, L20 
Luhman, K. L., Patten, B. M., Marengo, M., et al. 2007, ApJ, 654, 570

Marley, M. S., Saumon, D., Cushing, M., et al. 2012, ApJ, 754, 135

Marois, C., Macintosh, B., Barman, T., et al. 2008, Science, 322, 1348

Metchev, S. A. \& Hillenbrand, L. A. 2006, ApJ, 651, 1166

Naud, M.-E., Artigau, É., Malo, L., et al. 2014, ApJ, 787, 5

Oppenheimer, B. R., Baranec, C., Beichman, C., et al. 2013, ApJ, 768, 24

Patience, J., King, R. R., de Rosa, R. J., \& Marois, C. 2010, A\&\&A, 517, A76

\section{Discussion}

J. GaGné: You show some very red objects with "peaky" H-band spectra that are not young. Do you have an explanation for why these objects appear the way they do?

Author: There is some speculation in the literature that this could be a metallicity or inclination effect.

S. Metchev: Please get to the bottom of these dust vs. youth indicators. Also, how useful is $\mathrm{VO}$ as a youth indicator?

Author: In Allers \& Liu (2013), we present a classification system that can distinguish between young and dusty objects. As for VO, it is an excellent indicator of youth for objects with spectral types of L0-L4.

B. Bowler: Could some of the youth effects be caused by patchy clouds? i.e. could it be that gravity effects are actually caused by seeing emergent spectra from different cloud layers in the atmosphere?

Author: Patchy clouds could certainly do interesting things to the spectra (see contribution by M. Marley), but the youth that we see isn't likely driven by cloud patchiness. We see strong indicators of youth in objects with late $\mathrm{M}$ and early $\mathrm{L}$ spectral types which shouldn't have the patchy clouds that are associated with the L/T transition. 